Supporting Information for

\title{
Influence of Intermolecular Interactions on the Mössbauer Quadrupole Splitting of Organotin(IV) Compounds as Studied by DFT Calculations
}

Szilvia Kárpáti,,*, Roland Szalay, ${ }^{\ddagger}$ Attila G. Császár, ${ }^{\#}$ Károly Süvegh, ${ }^{\dagger}$ Sándor Nagy ${ }^{\dagger}$

Institute of Chemistry, Eötvös Loránd University, P.O.B 32 H-1518 Budapest 112, Hungary

\author{
${ }^{\dagger}$ Laboratory of Nuclear Chemistry \\ * Organosilicon Chemistry Laboratory \\ \# Laboratory of Molecular Spectroscopy
}


TABLE S1: Calculated EFG components $\left(V_{\mathrm{xx}}, V_{\mathrm{yy}}, V_{\mathrm{zz}}\right) V$ values in atomic units and quadrupole splittings $\left(\Delta_{\mathrm{calc}}\right)$ in $\mathrm{mm} \mathrm{s}^{-1}$ obtained at optimized or solid-state (non-optimized) geometries and at the central tin atom in supramolecular moieties of different numbers of molecules for compounds 1-5. The asymmetry parameter $(\eta)$, calculated $V$ values and quadrupole splittings are also shown.

\begin{tabular}{|c|c|c|c|c|c|c|}
\hline Supramolecular moiety of ${ }^{a}$ & $V_{\mathrm{xx}}$ & $V_{\mathrm{yy}}$ & $V_{\mathrm{zz}}$ & $\eta$ & $V$ & $\Delta_{\text {calc }}$ \\
\hline \multicolumn{7}{|l|}{ Compound 1: } \\
\hline 1 (gas phase, optimized) & -1.50 & -2.70 & 4.20 & 0.28 & 4.25 & 3.95 \\
\hline 1 (solid state, non-optimized) & -1.44 & -2.79 & 4.23 & 0.32 & 4.30 & 3.99 \\
\hline 3 & -1.44 & -2.80 & 4.24 & 0.32 & 4.32 & 4.01 \\
\hline 5 & -1.46 & -2.81 & 4.26 & 0.32 & 4.33 & 4.03 \\
\hline 7 & -1.43 & -2.86 & 4.29 & 0.33 & 4.37 & 4.06 \\
\hline 9 & -1.48 & -2.79 & 4.27 & 0.31 & 4.34 & 4.03 \\
\hline 13 & -1.42 & -2.84 & 4.26 & 0.33 & 4.34 & 4.04 \\
\hline \multicolumn{7}{|l|}{ Compound 2: } \\
\hline 1 (gas phase, optimized) & 0.88 & 0.95 & -1.83 & 0.04 & -1.83 & -1.70 \\
\hline 1 (solid state, non-optimized) & 0.98 & 1.02 & -2.00 & 0.02 & -2.00 & -1.86 \\
\hline 1 (solid state, non-optimized) & 0.83 & 0.84 & -1.67 & 0.01 & -1.66 & -1.55 \\
\hline 2 & 0.98 & 1.08 & -2.06 & 0.05 & -2.06 & -1.92 \\
\hline 3 & 0.98 & 1.07 & -2.05 & 0.04 & -2.05 & -1.91 \\
\hline 4 & 1.01 & 1.10 & -2.11 & 0.04 & -2.11 & -1.96 \\
\hline 6 & 0.97 & 1.16 & -2.13 & 0.09 & -2.13 & -1.98 \\
\hline 7 & 0.91 & 1.13 & -2.04 & 0.11 & -2.05 & -1.90 \\
\hline \multicolumn{7}{|l|}{ Compound 3: } \\
\hline 1 (gas phase, optimized) & 1.27 & 1.35 & -2.62 & 0.03 & -2.62 & -2.44 \\
\hline \multirow[t]{2}{*}{1 (solid state, non-optimized) } & 1.39 & 1.71 & -3.11 & 0.10 & -3.11 & -2.89 \\
\hline & 1.11 & 1.89 & -3.00 & 0.26 & -3.03 & -2.82 \\
\hline 2 & 1.56 & 1.70 & -3.27 & 0.04 & -3.27 & -3.04 \\
\hline 3 & 1.62 & 1.81 & -3.43 & 0.06 & -3.43 & -3.19 \\
\hline 4 & 1.61 & 1.87 & -3.48 & 0.075 & -3.49 & -3.24 \\
\hline 5 & 1.61 & 1.90 & -3.51 & 0.08 & -3.51 & -3.26 \\
\hline 6 & 1.62 & 1.89 & -3.514 & 0.07 & -3.52 & -3.27 \\
\hline \multicolumn{7}{|l|}{ Compound 4: } \\
\hline 1 (gas phase, optimized) & 1.28 & 1.30 & -2.58 & 0.01 & -2.58 & -2.40 \\
\hline 1 (solid state, non-optimized) & 1.29 & 1.53 & -2.82 & 0.08 & -2.83 & -2.63 \\
\hline 2 & 1.31 & 1.70 & -3.02 & 0.13 & -3.03 & -2.82 \\
\hline 3 & 1.33 & 1.79 & -3.12 & 0.15 & -3.13 & -2.91 \\
\hline 4 & 1.37 & 1.80 & -3.17 & 0.13 & -3.18 & -2.96 \\
\hline 12 & 1.43 & 1.77 & -3.20 & 0.10 & -3.21 & -2.98 \\
\hline \multicolumn{7}{|l|}{ Compound 5: } \\
\hline 1 (gas phase, optin & 1.17 & 1.17 & -2.33 & 0.00 & -2.33 & -2.17 \\
\hline 1 (solid state, non-optimized) & 1.32 & 1.38 & -2.71 & 0.02 & -2.71 & -2.52 \\
\hline 2 & 1.38 & 1.47 & -2.85 & 0.03 & -2.85 & -2.65 \\
\hline 3 & 1.58 & 1.66 & -3.25 & 0.02 & -3.25 & -3.02 \\
\hline 6 & 1.66 & 1.73 & -3.39 & 0.02 & -3.39 & -3.16 \\
\hline 9 & 1.65 & 1.87 & -3.52 & 0.06 & -3.52 & -3.28 \\
\hline 9 & 1.72 & 1.87 & -3.59 & 0.04 & -3.59 & -3.34 \\
\hline 27 & 1.63 & 1.83 & -3.47 & 0.06 & -3.47 & -3.23 \\
\hline
\end{tabular}

$a$ The number of molecules composing the supramolecular moiety considered (e.g., supramolecular moiety of 3 means a trimer). 
TABLE S2: Average $\mathrm{C}-\mathrm{Sn}-\mathrm{Cl}$ angles (in degrees) and $\mathrm{Sn}-\mathrm{Cl}$ bond distances (in $\AA$ ) of the $\mathrm{R}_{3} \mathrm{SnCl}$ compounds used for establishing the new correlation function given in this paper.

\begin{tabular}{|c|c|c|}
\hline $\begin{array}{l}\text { CSD code } \\
\text { (structure) }\end{array}$ & $\mathrm{C}-\mathrm{Sn}-\mathrm{Cl}$ & $\mathrm{d}(\mathrm{Sn}-\mathrm{Cl})$ \\
\hline $\mathrm{Ph}_{3} \mathrm{SnCl}_{2}^{-}$ & $90.01^{2}$ & $2.598^{2}$ \\
\hline JEYVEO & 93.14 & 2.514 \\
\hline LEPCEO & 93.45 & 2.508 \\
\hline BEJZIZ & 93.52 & 2.515 \\
\hline KUGTUB01 & 93.70 & 2.509 \\
\hline GUGKIC & 93.79 & 2.488 \\
\hline CAYLIX10 & 93.93 & 2.503 \\
\hline TERGOM & 93.96 & 2.482 \\
\hline YOVHIA & 94.03 & 2.476 \\
\hline CIYVOV & 94.43 & 2.485 \\
\hline BIVGUI & 95.03 & 2.484 \\
\hline HIFLUD & 95.04 & 2.491 \\
\hline PPECSN & 95.14 & 2.475 \\
\hline LEPCIS & 95.18 & 2.483 \\
\hline IBIRAM & 95.26 & 2.469 \\
\hline GUGKEY & 95.29 & 2.488 \\
\hline TIQWIZ & 95.39 & 2.459 \\
\hline FOGBEI & 95.39 & 2.485 \\
\hline KAWVIN & 95.40 & 2.484 \\
\hline KUGTUB & 95.49 & 2.470 \\
\hline KUGTUB02 & 95.49 & 2.476 \\
\hline UCADUX & 95.73 & 2.479 \\
\hline KUWNIZ & 95.76 & 2.457 \\
\hline GUGKOI & 95.85 & 2.483 \\
\hline RIHNIF & 95.91 & 2.451 \\
\hline YUCYIE & 96.97 & 2.451 \\
\hline WEJROS & 97.29 & 2.439 \\
\hline UBIFOA & 97.35 & 2.455 \\
\hline CTPHSN09 & 97.74 & 2.466 \\
\hline CTPHSN08 & 97.76 & 2.465 \\
\hline CTPHSN07 & 97.91 & 2.461 \\
\hline CTPHSN06 & 98.22 & 2.456 \\
\hline CTPHSN05 & 98.90 & 2.444 \\
\hline CTPHSN04 & 99.09 & 2.441 \\
\hline TMSNCL & 99.12 & 2.430 \\
\hline CTPHSN03 & 99.49 & 2.426 \\
\hline CTPHSN02 & 99.72 & 2.416 \\
\hline CTPHSN01 & 100.30 & 2.415 \\
\hline MUGGUQ & 101.82 & 2.390 \\
\hline MUGGUQ & 101.90 & 2.388 \\
\hline TERQIQ & 102.48 & 2.387 \\
\hline MUGGOK & 102.62 & 2.389 \\
\hline MUGGOK & 103.02 & 2.382 \\
\hline MUGGOK & 103.19 & 2.388 \\
\hline TPSNCL03_Sn2 & 103.97 & 2.374 \\
\hline ETALAM & 104.10 & 2.376 \\
\hline ETALEQ & 104.36 & 2.373 \\
\hline TPSNCL02_Sn2 & 104.39 & 2.364 \\
\hline NIQZUI & 104.41 & 2.379 \\
\hline TICCAJ & 104.49 & 2.346 \\
\hline TPSNCL01_Sn2 & 104.85 & 2.356 \\
\hline TPSNCL02_Sn1 & 105.01 & 2.357 \\
\hline TPSNCL01_Sn1 & 105.30 & 2.354 \\
\hline TPSNCL03_Sn1 & 105.88 & 2.353 \\
\hline HIBTAN & 106.29 & 2.349 \\
\hline
\end{tabular}


Optimized geometries (cartesian coordinates, in $\AA$ ) of complexes 1-5 described in the main text at the DFT(B3LYP) level of theory; computations carried out with the suite of programs Gaussian03. ${ }^{3}$

Optimized geometry of $\mathbf{1}$

\begin{tabular}{|c|c|c|c|c|}
\hline$\#$ & Symbol & $x$ & $Y$ & z \\
\hline 1 & $\mathrm{Sn}$ & 0.000042 & -0.000390 & 0.000011 \\
\hline 2 & C & -0.174418 & -0.001428 & -2.122139 \\
\hline 3 & $\mathrm{H}$ & 0.307542 & -0.892876 & -2.534064 \\
\hline 4 & H & -1.227481 & -0.000747 & -2.417587 \\
\hline 5 & H & 0.309019 & 0.888637 & -2.535329 \\
\hline 6 & c & 0.174244 & -0.001674 & 2.122181 \\
\hline 7 & H & 1.227250 & 0.000763 & 2.417831 \\
\hline 8 & H & -0.310843 & 0.887428 & 2.535505 \\
\hline 9 & H & -0.306224 & -0.894079 & 2.533779 \\
\hline 10 & 0 & -1.585617 & 1.476970 & 0.109208 \\
\hline 11 & 0 & 1.585592 & 1.476958 & -0.109095 \\
\hline 12 & 0 & -1.586857 & -1.477095 & 0.109183 \\
\hline 13 & 0 & 1.586870 & -1.477083 & -0.109233 \\
\hline 14 & C & 2.844218 & -1.258646 & -0.029434 \\
\hline 15 & C & 3.471365 & 0.000723 & 0.033484 \\
\hline 16 & c & 2.843177 & 1.259513 & -0.029320 \\
\hline 17 & c & -2.844205 & -1.258661 & 0.029349 \\
\hline 18 & c & -3.471358 & 0.000706 & -0.033544 \\
\hline 19 & C & -2.843183 & 1.259506 & 0.029353 \\
\hline 20 & $\mathrm{H}$ & -4.552804 & 0.001159 & -0.098679 \\
\hline 21 & H & 4.552813 & 0.001181 & 0.098576 \\
\hline 22 & C & -3.702063 & -2.505703 & 0.015074 \\
\hline 23 & H & -3.514091 & -3.082162 & 0.928130 \\
\hline 24 & $\mathrm{H}$ & -4.769827 & -2.285639 & -0.057862 \\
\hline 25 & $\mathrm{H}$ & -3.404042 & -3.138843 & -0.828331 \\
\hline 26 & c & -3.699947 & 2.507285 & 0.014964 \\
\hline 27 & H & -3.401970 & 3.139671 & -0.829030 \\
\hline 28 & $\mathrm{H}$ & -4.767951 & 2.288113 & -0.057127 \\
\hline 29 & H & -3.510816 & 3.084122 & 0.927538 \\
\hline 30 & c & 3.702066 & -2.505688 & -0.015167 \\
\hline 31 & H & 4.769859 & -2.285619 & 0.057318 \\
\hline 32 & $\mathrm{H}$ & 3.404361 & -3.138606 & 0.828521 \\
\hline 33 & $\mathrm{H}$ & 3.513730 & -3.082384 & -0.927996 \\
\hline 34 & c & 3.699912 & 2.507304 & -0.014948 \\
\hline 35 & H & 4.767910 & 2.288154 & 0.057297 \\
\hline 36 & $\mathrm{H}$ & 3.510885 & 3.084045 & -0.927603 \\
\hline 37 & H & 3.401819 & 3.139769 & 0.828946 \\
\hline
\end{tabular}

Optimized geometry of 2

\begin{tabular}{|c|c|c|c|c|}
\hline \# & Symbol & $x$ & $Y$ & z \\
\hline 1 & Sn & -0.005452 & -0.018031 & 0.476216 \\
\hline 2 & $\mathrm{Cl}$ & -0.055161 & -0.141599 & 2.853112 \\
\hline 3 & C & -0.265779 & 2.012046 & -0.103002 \\
\hline 4 & C & -1.513690 & 2.467803 & -0.560377 \\
\hline 5 & C & 0.803287 & 2.922906 & -0.046564 \\
\hline 6 & C & -1.690158 & 3.798428 & -0.946959 \\
\hline 7 & $\mathrm{H}$ & -2.357729 & 1.784006 & -0.620152 \\
\hline 8 & C & 0.628199 & 4.253234 & -0.433197 \\
\hline 9 & $\mathrm{H}$ & 1.783056 & 2.598723 & 0.297650 \\
\hline 10 & C & -0.619273 & 4.691843 & -0.882734 \\
\hline 11 & $\mathrm{H}$ & -2.661775 & 4.136443 & -1.298149 \\
\hline 12 & H & 1.464011 & 4.946429 & -0.383153 \\
\hline 13 & H & -0.755646 & 5.727408 & -1.183496 \\
\hline 14 & C & -1.617417 & -1.240976 & -0.183918 \\
\hline 15 & C & -1.459139 & -2.031823 & -1.334439 \\
\hline 16 & C & -2.854826 & -1.260335 & 0.481190 \\
\hline 17 & C & -2.511850 & -2.815643 & -1.813120 \\
\hline 18 & $\mathrm{H}$ & -0.507027 & -2.048519 & -1.861473 \\
\hline 19 & C & -3.907881 & -2.043551 & 0.003180 \\
\hline 20 & $\mathrm{H}$ & -2.999846 & -0.675145 & 1.386462 \\
\hline 21 & C & -3.737637 & -2.820167 & -1.145079 \\
\hline 22 & $\mathrm{H}$ & -2.373121 & -3.424815 & -2.702683 \\
\hline 23 & H & -4.858857 & -2.051061 & 0.529679 \\
\hline 24 & $\mathrm{H}$ & -4.556983 & -3.430961 & -1.515261 \\
\hline 25 & C & 1.903404 & -0.745230 & -0.115263 \\
\hline 26 & C & 2.601030 & -1.691545 & 0.653465 \\
\hline 27 & C & 2.476222 & -0.302171 & -1.319927 \\
\hline 28 & C & 3.835491 & -2.185171 & 0.226416 \\
\hline 29 & H & 2.187474 & -2.039803 & 1.596946 \\
\hline 30 & C & 3.710259 & -0.797882 & -1.749299 \\
\hline 31 & $\mathrm{H}$ & 1.968502 & 0.443432 & -1.929041 \\
\hline 32 & C & 4.389863 & -1.740555 & -0.976095 \\
\hline 33 & $\mathrm{H}$ & 4.365534 & -2.914607 & 0.833463 \\
\hline 34 & H & 4.141221 & -0.444711 & -2.682719 \\
\hline 35 & $\mathrm{H}$ & 5.350912 & -2.125317 & -1.307460 \\
\hline
\end{tabular}


Optimized geometry of $\mathbf{3}$

\begin{tabular}{|c|c|c|c|c|}
\hline$\#$ & Symbol & $\mathrm{X}$ & $\mathrm{Y}$ & $\mathrm{Z}$ \\
\hline 1 & $\mathrm{Sn}$ & 1.123763 & 0.015359 & 0.000067 \\
\hline 2 & $\mathrm{O}$ & -1.467018 & 2.320345 & 0.003515 \\
\hline 3 & $\mathrm{O}$ & -1.155575 & -2.246606 & 0.003559 \\
\hline 4 & $\mathrm{~N}$ & -0.984106 & 0.052428 & -0.004899 \\
\hline 5 & $\mathrm{C}$ & 1.637052 & -0.977088 & 1.822502 \\
\hline 6 & $\mathrm{C}$ & 1.778234 & 2.047503 & -0.060681 \\
\hline 7 & $\mathrm{C}$ & 1.649148 & -1.084801 & -1.755682 \\
\hline 8 & $\mathrm{C}$ & -1.684019 & -1.146735 & -0.001703 \\
\hline 9 & $\mathrm{C}$ & -3.186876 & -0.859811 & -0.004665 \\
\hline 10 & $\mathrm{C}$ & -3.284878 & 0.671632 & -0.002772 \\
\hline 11 & $\mathrm{C}$ & -1.830345 & 1.159847 & -0.001286 \\
\hline 12 & $\mathrm{H}$ & -3.635475 & -1.329337 & -0.886029 \\
\hline 13 & $\mathrm{H}$ & -3.639839 & -1.332269 & 0.872824 \\
\hline 14 & $\mathrm{H}$ & -3.792647 & 1.081551 & -0.881654 \\
\hline 15 & $\mathrm{H}$ & -3.793347 & 1.079109 & 0.876839 \\
\hline 16 & $\mathrm{H}$ & 2.870468 & 2.093231 & 0.009173 \\
\hline 17 & $\mathrm{H}$ & 1.342205 & 2.613945 & 0.765366 \\
\hline 18 & $\mathrm{H}$ & 1.463786 & 2.522760 & -0.993452 \\
\hline 19 & $\mathrm{H}$ & 1.146567 & -2.055057 & -1.733962 \\
\hline 20 & $\mathrm{H}$ & 2.731042 & -1.245892 & -1.805564 \\
\hline 21 & $\mathrm{H}$ & 1.340935 & -0.546672 & -2.657516 \\
\hline 22 & $\mathrm{H}$ & 2.717344 & -1.144874 & 1.883708 \\
\hline 23 & $\mathrm{H}$ & 1.332922 & -0.381321 & 2.688708 \\
\hline 24 & $\mathrm{H}$ & 1.127480 & -1.943320 & 1.861627 \\
\hline & & & & \\
\hline 17 & & & \\
\hline & & & \\
\hline & & &
\end{tabular}

Optimized geometry of $\mathbf{4}$

\begin{tabular}{|c|c|c|c|c|}
\hline$\#$ & Symbol & $\mathrm{X}$ & $\mathrm{Y}$ & $\mathrm{Z}$ \\
\hline 1 & $\mathrm{Sn}$ & -0.000030 & 1.957131 & 0.000000 \\
\hline 2 & $\mathrm{O}$ & 0.031054 & -0.528658 & 2.299896 \\
\hline 3 & $\mathrm{O}$ & 0.031054 & -0.528658 & -2.299896 \\
\hline 4 & $\mathrm{~N}$ & -0.033324 & -0.143123 & 0.000000 \\
\hline 5 & $\mathrm{C}$ & -0.006118 & -0.941421 & 1.152997 \\
\hline 6 & $\mathrm{C}$ & -0.006118 & -0.941421 & -1.152997 \\
\hline 7 & $\mathrm{C}$ & -0.013621 & -2.366869 & 0.697165 \\
\hline 8 & $\mathrm{C}$ & -0.013621 & -2.366869 & -0.697165 \\
\hline 9 & $\mathrm{C}$ & -0.011034 & -3.549168 & 1.424323 \\
\hline 10 & $\mathrm{C}$ & -0.011977 & -4.749200 & 0.701002 \\
\hline 11 & $\mathrm{C}$ & -0.011977 & -4.749200 & -0.701002 \\
\hline 12 & $\mathrm{C}$ & -0.011034 & -3.549168 & -1.424323 \\
\hline 13 & $\mathrm{C}$ & -1.024199 & 2.572996 & -1.770931 \\
\hline 14 & $\mathrm{C}$ & 2.079965 & 2.459032 & 0.000000 \\
\hline 15 & $\mathrm{C}$ & -1.024199 & 2.572996 & 1.770931 \\
\hline 16 & $\mathrm{H}$ & -0.008380 & -3.537005 & -2.509979 \\
\hline 17 & $\mathrm{H}$ & -0.011635 & -5.696670 & -1.232831 \\
\hline 18 & $\mathrm{H}$ & -0.011635 & -5.696670 & 1.232831 \\
\hline 19 & $\mathrm{H}$ & -0.008380 & -3.537005 & 2.509979 \\
\hline 20 & $\mathrm{H}$ & -0.566759 & 2.108810 & 2.646684 \\
\hline 21 & $\mathrm{H}$ & -2.074195 & 2.267220 & -1.728536 \\
\hline 22 & $\mathrm{H}$ & 2.570187 & 2.049013 & 0.888177 \\
\hline 23 & $\mathrm{H}$ & -0.987616 & 3.662760 & 1.873352 \\
\hline 24 & $\mathrm{H}$ & -2.074195 & 2.267220 & 1.728536 \\
\hline 25 & $\mathrm{H}$ & -0.987616 & 3.662760 & -1.873352 \\
\hline 26 & $\mathrm{H}$ & -0.566759 & 2.108810 & -2.646684 \\
\hline 27 & $\mathrm{H}$ & 2.570187 & 2.049013 & -0.888177 \\
\hline 28 & $\mathrm{H}$ & 2.218294 & 3.545304 & 0.000000 \\
\hline & & & & \\
\hline
\end{tabular}

Optimized geometry of $\mathbf{5}$

\begin{tabular}{|c|c|c|c|c|}
\hline$\#$ & Symbol & $\mathrm{X}$ & $\mathrm{Y}$ & $\mathrm{Z}$ \\
\hline 1 & $\mathrm{Sn}$ & 0.000000 & 0.000000 & 0.257781 \\
\hline 2 & $\mathrm{C}$ & 0.000000 & 2.058816 & 0.829271 \\
\hline 3 & $\mathrm{H}$ & 0.887268 & 2.563856 & 0.437032 \\
\hline 4 & $\mathrm{H}$ & -0.887268 & 2.563856 & 0.437032 \\
\hline 5 & $\mathrm{H}$ & 0.000000 & 2.152698 & 1.920688 \\
\hline 6 & $\mathrm{C}$ & -1.782987 & -1.029408 & 0.829271 \\
\hline 7 & $\mathrm{H}$ & -2.663998 & -0.513531 & 0.437032 \\
\hline 8 & $\mathrm{H}$ & -1.776730 & -2.050325 & 0.437032 \\
\hline 9 & $\mathrm{H}$ & -1.864291 & -1.076349 & 1.920688 \\
\hline 10 & $\mathrm{C}$ & 1.782987 & -1.029408 & 0.829271 \\
\hline 11 & $\mathrm{H}$ & 1.776730 & -2.050325 & 0.437032 \\
\hline 12 & $\mathrm{H}$ & 2.663998 & -0.513531 & 0.437032 \\
\hline 13 & $\mathrm{H}$ & 1.864291 & -1.076349 & 1.920688 \\
\hline 14 & $\mathrm{Cl}$ & 0.000000 & 0.000000 & -2.129424 \\
\hline
\end{tabular}




\section{References}

(1) Cambridge Crystallographic Structural Database version 5.27, November 2005.

(2) S. Weng Ng, Acta Crystallogr. Sect. A 1995, 51, 1124-1125.

(3) Gaussian 03, Revision C.02, Frisch, M. J.; Trucks, G. W.; Schlegel, H. B.; Scuseria, G. E.; Robb, M. A.; Cheeseman, J. R.; Montgomery, Jr., J. A.; Vreven, T.; Kudin, K. N.; Burant, J. C.; Millam, J. M.; Iyengar, S. S.; Tomasi, J.; Barone, V.; Mennucci, B.; Cossi, M.; Scalmani, G.; Rega, N.; Petersson, G. A.; Nakatsuji, H.; Hada, M.; Ehara, M.; Toyota, K.; Fukuda, R.; Hasegawa, J.; Ishida, M.; Nakajima, T.; Honda, Y.; Kitao, O.; Nakai, H.; Klene, M.; Li, X.; Knox, J. E.; Hratchian, H. P.; Cross, J. B.; Bakken, V.; Adamo, C.; Jaramillo, J.; Gomperts, R.; Stratmann, R. E.; Yazyev, O.; Austin, A. J.; Cammi, R.; Pomelli, C.; Ochterski, J. W.; Ayala, P. Y.; Morokuma, K.; Voth, G. A.; Salvador, P.; Dannenberg, J. J.; Zakrzewski, V. G.; Dapprich, S.; Daniels, A. D.; Strain, M. C.; Farkas, O.; Malick, D. K.; Rabuck, A. D.; Raghavachari, K.; Foresman, J. B.; Ortiz, J. V.; Cui, Q.; Baboul, A. G.; Clifford, S.; Cioslowski, J.; Stefanov, B. B.; Liu, G.; Liashenko, A.; Piskorz, P.; Komaromi, I.; Martin, R. L.; Fox, D. J.; Keith, T.; Al-Laham, M. A.; Peng, C. Y.; Nanayakkara, A.; Challacombe, M.; Gill, P. M. W.; Johnson, B.; Chen, W.; Wong, M. W.; Gonzalez, C.; and Pople, J. A.; Gaussian, Inc., Wallingford CT, 2004. 